\title{
Quantities and Units for Use in Radiation Protection
}

\author{
A Draft Report
}

\author{
A. Allisy, W.A. Jennings, A.M. Kellerer, J.W. Müller, and H.H. Rossi \\ ICRU \\ Bethesda, MD 20814-3095, U.S.A.
}

\section{Introduction}

The International Conmission on Radiation Units and Measurements asked its Committee on Fundamental Quantities and Units to revise ICRU Report 33 (ICRU, 1980), entitled "Radiation Quantities and Units". Report 33 is divided into two parts, $A$ and $B$. Part A deals with quantities and units of general use. Part $B$ concerns quantities and units for use in radiation protection. Since ICRU Report 33 was published, a number of discussions have taken place, especially in the field of quantities for use in radiation protection measurements. The results of these discussions have been published in ICRU Reports 39 (ICRU, 1985) and 43 (ICRU, 1988), where operational quantities have been introduced for the specification of the dose equivalents for environmental and individual monitoring in the case of external radiation sources. The International Commission on Radiological Protection (ICRP), in its 1990 Recommendations (ICRP, 1991), has introduced radiation weighting factors, and two consequential new quantities, equivalent organ dose and effective dose. The radiation weighting factors, applicable to the mean absorbed dose in a tissue or organ, depend only on the type and energy of the radiation incident on the body in the case of external irradiation. They cannot be defined for mixed radiations and are inappropriate for measurement purposes, such as area monitoring. These new developments in this field led the members of the Committee on Fundamental Quantities and Units to concentrate their effort first toward the revision of part $B$ of Report 33. As a result, the present draft has been produced; it is published under the names of the Committee Members who invite and welcome constructive comments on their work (see the Chairman's page).

\section{General Considerations}

The determination of quantities relevant to radiation protection often involve significant uncertainties. In addition, a variety of approximations must be used for relating physical measurements to biological effects caused by radiation. Although a comparatively wide margin may be admissible in radiation protection, it is essential that the quantities employed be unambiguously defined and that the approximations be clearly identified.

The basic radiation protection quantities are defined in this Report. They are used by the International Commission on Radiological Protection (ICRP) to define further quantities, and publications of ICRP should be consulted for their definitions.

The biological effect of a given absorbed dose of ionizing radiation depends on the microscopic distribution of the absorbed energy in the irradiated tissue. The quality factor, $Q$, weights the absorbed dose for the biological effectiveness of the charged particles producing the absorbed dose. It is formulated to take account of the relative effectiveness of the different types of ionizing radiation at the low exposure levels encountered in routine radiation protection practice.

$Q$ can be defined as a function of linear energy transfer, $L$, in tissue or water by a relation of the form

$$
Q=(1 / D) \int_{L} Q(L) D_{L} \mathrm{~d} L,
$$

where $D$ is the absorbed dose delivered at a point and $D_{L} \mathrm{~d} L$ is that part of the absorbed dose delivered by particles with (unrestricted) linear energy transfers between $L$ and $L+\mathrm{d} L$ (ICRU, 1970). The integration is to be performed over the distribution of $L$ from all charged particles excluding their secondary electrons.

$D_{L}$ equals $\phi_{L} L / \rho$, where $\phi_{L}$ is the spectral distribution of the charged particle fluence (except secondary electrons), and $\rho$ is the density of the reference material.

A functional dependence of $Q$ in terms of the unrestricted linear energy transfer, $L$, in water has been specified by ICRP (ICRP, 1991), as follows :

$$
Q(L)= \begin{cases}1 & \text { for } L \leq 10, \\ 0.32 L-2.2 & \text { for } 10<L<100, \\ 300 / \sqrt{L} & \text { for } L \geq 100\end{cases}
$$

where $L$ is expressed in $\mathrm{keV} \mu \mathrm{m}^{-1}$.

The subject of $Q$ has been considered in a report of a Joint Task Group of the ICRP and the ICRU. In that report (ICRU, 1986), it is concluded that, for purposes of 
radiation protection, radiation quality should be specified in terms of lineal energy, $y$, in a $1 \mu \mathrm{m}$ diameter sphere of ICRU tissue (muscle). $Q$ can then be defined by a relation of the form

$$
Q=(1 / D) \int_{y} Q(y) D_{y} \mathrm{~d} y,
$$

where $D_{y}$ dy is the absorbed dose delivered by particles with lineal energies between $y$ and $y+\mathrm{d} y$ (ICRU, 1983). The value of $Q$ obtained using the above relation may deviate slightly from the value obtained as a function of $L$, depending on the choice of the cut off energy of the secondary electrons.

\section{Dose Equivalent}

For routine radiation-protection applications, a quantity called dose equivalent is defined. It should not be used in numerical assessment of high-level, accidental exposures (ICRP, 1977).

\subsection{Dose Equivalent at a Point}

The dose equivalent, $H$, is the product of $Q$ and $D$ at a point in tissue, where $D$ is the absorbed dose and $Q$ the quality factor at that point, thus

$$
H=Q D \text {. }
$$

Unit: $\mathrm{J}_{\mathrm{g}}{ }^{-1}$

The special name for the unit of dose equivalent is sievert (Sv).

Previous definitions of $H$ included an additional factor, $N$, as a product of other modifying factors. Its deletion was recommended by the Joint Task Group of the ICRP and ICRU (ICRU, 1986) and by ICRP (ICRP, 1991).

\subsection{Dose Equivalent Rate}

The dose equivalent rate, $H$, is the quotient of $\mathrm{d} H$ by dt, where dH is the increment of dose equivalent in the time interval ot.

$$
H=\mathrm{d} H \boldsymbol{\mathrm { d }} \mathrm{s}
$$

Unit: $\operatorname{~skg-1~}^{-1}$

The special name for the unit of dose equivalent rate is stevert per second $\left(\mathrm{Sv}^{-1}\right)$.
Analogous rate quantities can be defined for other dosimetric quantities used in radiation protection applications.

\section{Quantities Based on Mean Values}

Because $D$ and $Q$ depend on the location in the body and on its orientation in the radiation field, the following three mean-value quantities averaged over an extended mass must be formulated as integrals.

\subsection{Mean Absorbed Dose in an Organ}

The mean absorbed dose, $D_{\mathrm{T}}$, in a specified tissue T or organ, is given by

$$
D_{\mathrm{T}}=\left(1 / m_{\mathrm{T}}\right) \int_{m_{\mathrm{T}}} D \mathrm{~d} m,
$$

where $m_{\mathrm{T}}$ is the mass of the tissue or organ, and $D$ is the absorbed dose in the mass element $\mathrm{d} m$.

The mean absorbed dose, $D_{\mathrm{T}}$, in a specified tissue or organ, equals the ratio of the mean energy imparted, $\epsilon_{T}$, to the tissue or organ and $m_{\mathrm{T}}$, the mass of the tissue or organ.

In the case of irradiations from external sources, the mean absorbed dose in a specified tissue or organ depends on the ambient radiation field, on the size and orientation of the body in this field, and on the organ.

The mean absorbed dose in a specified organ is sometimes termed the organ dose.

\subsection{Mean Quality Factor in an Organ}

The mean quality factor, $Q_{\mathrm{T}}$, in a specified tissue or organ, is given by

$$
Q_{\mathrm{T}}=\left(1 / m_{\mathrm{T}} D_{\mathrm{T}}\right) \int_{m_{\mathrm{T}}} Q D \mathrm{~d} m,
$$

where $D_{\mathrm{T}}$ is the mean absorbed dose to the tissue or organ, $m_{\mathrm{T}}$ is its mass, and $Q$ and $D$ are the quality factor and the absorbed dose in the mass element $\mathrm{d} m$, respectively.

In the case of irradiations from external sources, the mean quality factor in a specified tissue or organ depends on the ambient radiation field, on the size and orientation of the body in this field, and on the organ. 
The mean quality factor in a specified organ is sometimes termed the organ quality factor.

The ICRP (ICRP, 1991) has formulated radiation weighting factors, $w_{R}$, and specified numerical values in terms of particle type and energy range. These specifications apply to the radiations emitted in the case of internal irradiation; in connection with external irradiation, they refer to the radiation incident on the body and they do not depend on location in, or orientation of, the body. They should therefore not be confused with $Q_{\mathrm{T}}$.

\subsection{Mean Dose Equivalent in an Organ}

The mean dose equivalent, $H_{\mathrm{T}}$, in a specified tissue or organ, is given by

$$
H_{\mathrm{T}}=Q_{\mathrm{T}} D_{\mathrm{T}} \text {, }
$$

where $Q_{\mathrm{T}}$ is the mean quality factor in the organ and $D_{\mathrm{T}}$ is the mean absorbed dose to the organ.

The mean dose equivalent, $H_{\mathrm{T}}$, in a specified tissue or organ, can be expressed in terms of $L$ or $y$ distributions of the point functions $Q$ and $D$, thus

or

$$
H_{\mathrm{T}}=\left(1 / m_{\mathrm{T}}\right) \int_{m_{\mathrm{T}}} \int_{L} Q(L) D_{L} \mathrm{~d} L \mathrm{~d} m
$$

$$
H_{\mathrm{T}}=\left(1 / m_{\mathrm{T}}\right) \int_{m_{\mathrm{T}}} \int_{y} Q(y) D_{y} \mathrm{~d} y \mathrm{~d} m .
$$

The mean dose equivalent to a specified organ is sometimes termed the organ dose equivalent.

The ICRP (ICRP, 1991) has introduced the equivalent organ dose. It equals the sum of the organ doses due to the component radiations, $R$, weighted by the corresponding values of $w_{R}$.

The equivalent organ dose is often approximately equal to the organ dose equivalent, but the two concepts should not be confused, despite the similarity of the names.

The radiation weighting factor for a radiation with different components is the sum of the radiation weighting factors, $w_{R}$, of the component radiations, $R$, weighted according to their relative contributions to the organ dose. The resulting factor thus depends on the directional distribution of the radiation field, on the size and orientation of the body, and on the organ.

The individual contributions of the components of the ambient radiation field to the organ doses are needed for the determination of the equivalent organ dose, but these contributions cannot be separated in measurements. Therefore, radiation weighting factors cannot be employed in measurements. For measurement purposes, quantities defined in terms of $Q$, namely dose equivalent, ambient dose equivalent, directional dose equivalent and personal dose equivalent (see Section 6 ), are to be used.

\section{Effective Dose Equivalent}

The ICRP (ICRP, 1977) has defined a whole-body dose equivalent, termed the effective dose equivalent, $H_{\mathrm{E}}$, where

$$
H_{\mathrm{E}}=\sum_{\mathrm{T}} w_{\mathrm{T}} H_{\mathrm{T}}, \quad \text { with } \quad \sum_{\mathrm{T}} w_{\mathrm{T}}=1,
$$

and has specified the numerical values of the organ weighting factors, $w_{\mathrm{T}}$, for relevant organs or tissues, $T$. Current values are given in Table 1 (ICRP, 1991).

Table 1: Tissue-weighting factors

Tissue or organ Tissue weighting factor, $w_{\mathrm{T}}$

$\begin{array}{ll}\text { Gonads } & 0.20 \\ \text { Bone marrow (red) } & 0.12 \\ \text { Colon } & 0.12 \\ \text { Lung } & 0.12 \\ \text { Stomach } & 0.12 \\ \text { Bladder } & 0.05 \\ \text { Breast } & 0.05 \\ \text { Liver } & 0.05 \\ \text { Oesophagus } & 0.05 \\ \text { Thyroid } & 0.05 \\ \text { Skin } & 0.01 \\ \text { Bone surface } & 0.01 \\ \text { Remainder } & 0.05\end{array}$

The operational quantities, ambient dose equivalent, directional dose equivalent and personal dose equivalent (see Section 6) have been developed as measurable quantities related to the effective dose equivalent.

The ICRP (ICRP, 1991) has introduced a modified quantity that it terms effective dose which is equal to the sum of the equivalent organ doses weighted by the organ weighting factors. This quantity is often approximately equal to the effective dose equivalent. For measurement purposes the operational quantities are deemed appropriate.

\section{Operational Quantities for Whole-body Irradiation from External Sources}

Operational quantities have been devised for practical measurements, for both area and individual monitoring.

For purposes of routine radiation protection, it is desirable to characterize, in unoccupied space, the potential 
irradiation of individuals in terms of a single dose equivalent quantity that would exist in a phantom approximating the human body. The phantom selected is called the ICRU sphere. This ICRU sphere (ICRU, 1980) is a $30-\mathrm{cm}$ diameter tissue-equivalent sphere with a density of $1 \mathrm{~g} \mathrm{~cm}^{-3}$ and a mass composition of $76.2 \%$ oxygen, $11.1 \%$ carbon, $10.1 \%$ hydrogen and $2.6 \%$ nitrogen. In practical measurements, materials and elemental compositions with values differing somewhat from those specified above may provide adequate accuracy.

\subsection{Area Monitoring}

For area monitoring, it is useful to stipulate certain radiation fields that are derived from the actual radiation field. The terms "expanded" and "aligned" are used in this Report to characterize these derived radiation fields. In the expanded field, the fluence and its angular and energy distributions have the same value throughout the volume of interest as in the actual field at the point of reference. In the expanded and aligned field, the fluence and its energy distribution are the same as in the expanded field, but the fluence is unidirectional.

Radiations can be characterized as either weakly or strongly penetrating, depending on whether the dose equivalent, as a fraction of the relevant dose limits, is greater (a) for one of the organs, lens of the eye or skin, or (b) for the effective dose equivalent.

Two quantities linking the external radiation field to the effective dose equivalent and to the dose equivalent in the skin, and the lens of the eye have been introduced (ICRU, 1985) for purposes of area monitoring. These quantities are the ambient dose equivalent, $H^{*}(d)$, and the directional dose equivalent, $H^{\prime}(d, \Omega)$.

\subsubsection{Ambient Dose Equivalent, $H^{*}(d)$}

The ambient dose equivalent, $H^{*}(d)$, at a point in a radiation field, is the dose equivalent that would be produced by the corresponding expanded and aligned field in the ICRU sphere at a depth, $d$, on the radius opposing the direction of the aligned field.

Unit: $\mathrm{J} \mathrm{kg}^{-1}$

The special name for the unit of ambient dose equivalent is sievert (Sv).

Notes: a) Any statement of ambient dose equivalent should include a specification of the reference depth, $d$. In order to simplify notation, $d$ should be expressed in $\mathbf{m m}$. b) A quantity analogous to ambient dose equivalent can be defined in terms of absorbed dose. It is called ambient absorbed dose, $D^{*}(d)$.

For strongly penetrating radiation, a depth of $10 \mathrm{~mm}$ is frequently employed. The ambient dose equivalent for that depth is then denoted by $H^{*}(10)$. For weakly penetrating radiation, a depth of $0.07 \mathrm{~mm}$ for the skin and $3 \mathrm{~mm}$ for the eye are employed with analogous notations.

Measurement of $H^{*}(d)$ requires that the radiation field be uniform over the dimensions of the instrument and that the instrument have an isotropic response.

\subsubsection{Directional Dose Equivalent, $H^{\prime}(d, \Omega)$}

The directional dose equivalent $H^{\prime}(d, \Omega)$ at a point in a radiation field, is the dose equivalent that would. be produced by the corresponding expanded field in the ICRU sphere at a depth, $d$, on a radius in a specified direction, $\Omega$.

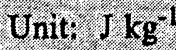

The special name for the unit of directional dose equivalent is sievert (Sv).

Notes: a) Any statement of directional dose equivalent should include a specification of the reference depth, $d$, and the direction $\Omega$. In order to simplify notation, $d$ should be expressed in $\mathrm{mm}$.

b) A quantity analogous to directional dose equivalent can be defined in terms of absorbed dose. It is called directional absorbed dose, $D^{\prime}(d, \Omega)$.

For weakly penetrating radiation, a depth of $0.07 \mathrm{~mm}$ for the skin and $3 \mathrm{~mm}$ for the eye are employed. The directional dose equivalent for these depths is then denoted by $H^{\prime}(0.07, \Omega)$ and $H^{\prime}(3, \Omega)$. For strongly penetrating radiation, a depth of $10 \mathrm{~mm}$ is frequently employed, with analogous notation.

Measurement of $H^{\prime}(d, \Omega)$ requires that the radiation field be uniform over the dimensions of the instrument, and that the instrument have the required directional response.

The specification of the direction $\Omega$ requires the choice of a reference system of coordinates, in which the direction $\Omega$ is expressed (e. g., polar and azimuthal angles). This reference system can often be related to the radiation field. In the particular case of a unidirectional field, the direction can be specified in terms of the angle, $\alpha$, between the 
radius opposing the incident field and the specified radius. When $\alpha=0$, the quantity $H^{\prime}(d, 0)$ may be written $H^{\prime}(d)$ and is equal to $H^{*}(d)$.

For weakly penetrating radiation, an instrument which determines the dose equivalent at the appropriate depth in a plane slab of tissue-equivalent material will adequately determine $H^{\prime}(0.07, \Omega)$ and also $H^{\prime}(3, \Omega)$ if the slab surface is perpendicular to $\Omega$.

\subsection{Individual Monitoring}

Instead of the two quantities in ICRU Report 39 (ICRU, 1985) for individual monitoring, a simplified concept, called personal dose equivalent, $H_{\mathrm{p}}(d)$, is introduced that is appropriate for both strongly and weakly penetrating radiations, depending on the value of $d$.

\subsubsection{Personal Dose Equivalent, $H_{\mathrm{p}}(d)$}

The personal dose equivalent, $H_{\mathrm{p}}(d)$, is the dose equivalent in soft tissue below a specified point on the body at an appropriate depth, $d$.

Unit: $\mathrm{J} \mathrm{kg}^{-1}$

The special name for the unit of personal dose equivalent is sievert (Sv).

Notes: a) Any statement of personal dose equivalent should include a specification of the reference depth, $d$. In order to simplify notation, $d$ should be expressed in $\mathrm{mm}$.

b) A quantity analogous to personal dose equivalent can be defined in terms of absorbed dose. It is called personal absorbed dose, $D_{\mathrm{p}}(d)$.

For weakly penetrating radiation, a depth of $0.07 \mathrm{~mm}$ for the skin and $3 \mathrm{~mm}$ for the eye are employed. The personal dose equivalent for those depths is then denoted by $H_{\mathrm{p}}(0.07)$ and $H_{\mathrm{p}}(3)$, respectively. For strongly penetrating radiation, a depth of $1.0 \mathrm{~mm}$ is frequently employed, with analogous notation.

$H_{\mathrm{p}}(d)$ can be measured with a detector which is worn at the surface of the body and covered with an appropriate thickness of tissue-equivalent material.

The calibration of the dosimeter is generally performed under simplified conditions on an appropriate phantom (ICRU, 1992).

\section{References}

ICRP (1977). International Commission on Radiological Protection, Recommendations of the ICRP, Publication 26, Annals of the ICRP 1 No. 3 (Pergamon Press, New York).

ICRP (1991). International Commission on Radiological Protection, 1990 Recommendations of the ICRP, Publication 60, Annals of the ICRP 21 No. 1-3 (Pergamon Press, New York).

ICRU (1970). International Commission on Radiation Units and Measurements, Linear Energy Transfer, ICRU Report 16 (International Commission on Radiation Units and Measurements, Bethesda, MD).

ICRU (1980). International Commission on Radiation Units and Measurements, Radiation Quantities and Units, ICRU Report 33 (International Commission on Radiation Units and Measurements, Bethesda, MD).

ICRU (1983). International Commission on Radiation Units and Measurements, Microdosimetry, ICRU Report 36 (International Commission on Radiation Units and Measurements, Bethesda, MD).

ICRU (1985). International Commission on Radiation Units and Measurements, Determination of Dose Equivalents Resulting from External Radiation Sources, ICRU Report 39 (International Commission on Radiation Units and Measurements, Bethesda, MD).

ICRU (1986). International Commission on Radiation Units and Measurements, The Quality Factor in Radiation Protection, ICRU Report 40 (International Commission on Radiation Units and Measurements, Bethesda, MD).

ICRU (1988). International Commission on Radiation Units and Measurements, Determination of Dose Equivalents from External Radiation Sources - Part 2, ICRU Report 43 (International Commission on Radiation Units and Measurements, Bethesda, MD).

ICRU (1992). International Commission on Radiation Units and Measurements, Measurement of Dose Equivalents from External Photon and Electron Radiations, ICRU Report 47 in press (International Commission on Radiation Units and Measurements, Bethesda, MD). 